GANIT J. Bangladesh Math. Soc. (ISSN 1606-3694) 39 (2019) 63-70

DOI: https://doi.org/10.3329/ganit.v39i0.44162

\title{
ON ORTHOGONAL GENERALIZED DERIVATIONS OF SEMIPRIME $\Gamma$-RINGS
}

\author{
${ }^{1}$ Kalyan Kumar Dey, ${ }^{2}$ Sanjay Kumar Saha and ${ }^{3}$ Akhil Chandra Paul \\ ${ }^{1,3}$ Department of Mathematics, Rajshahi University, Rajshahi-6205, Bangladesh \\ ${ }^{2}$ Department of Mathematics, Mawlana Bhasani Science and Technology University, \\ Tangail-1902, Bangladesh \\ ${ }^{1}$ kkdmath@yahoo.com, ${ }^{2}$ sanjaysahadu@gmail.com, ${ }^{3}$ acpaulru_math@yahoo.com
}

Received on 25-04-2018 Accepted on 11-11-2019

\begin{abstract}
In this paper, we study the orthogonality of two generalized derivations in semiprime $\Gamma$-rings. Some results are obtained in connection with ideals of semiprime $\Gamma$-rings and using left annihilator which is taken to be zero.
\end{abstract}

Keywords: Derivation, Generalized derivation, Orthogonal generalized derivation, Semiprime $\Gamma$-ring, $\Gamma$-ideal.

\section{Introduction}

Many mathematicians worked on derivations and generalized derivations of $\Gamma$-rings from several years. They obtained some remarkable results of prime and semiprime $\Gamma$-rings by using derivations. Their results enrich the field of algebra and modern analysis.

Ashraf and Jamal [3] studied on orthogonal derivations in $\Gamma$-rings. They obtained some necessary and sufficient conditions for the orthogonality of two derivations.

Suliman and Majeed [15] extended the results of [3] and studied the orthogonal derivations for a nonzero ideal of a semiprime gamma ring. Their results are related to some results concerning the product derivations on gamma rings.

Bresar and Vukman [6] initiated the study of orthogonal derivations in rings. Some results on orthogonal derivations in semiprime rings have been obtained by them related to product derivations.

The study of generalized derivations in rings was initiated by Hvala [13]. He obtained some fruitful results in rings with generalized derivations.

In this paper, we obtain some results of generalized derivations concerning the orthogonality in $\Gamma$ rings. The results which are obtained by us are the generalization of the results of Albas [1].

(C) GANIT: Journal of Bangladesh Mathematical Society, 2019 


\section{Preliminaries}

Let $M$ and $\Gamma$ be additive abelian groups. $M$ is called a $\Gamma$-ring if for all $a, b, c \in M, \alpha, \beta \in \Gamma$ the following conditions are satisfied :

(i) $a \beta b \in M$,

(ii) $(a+b) \alpha c=a \alpha c+b \alpha c, a(\alpha+\beta) b=a \alpha b+a \beta b, a \alpha(b+c)=a \alpha b+a \alpha c$,

(iii) $(a \alpha b) \beta c=a \alpha(b \beta c)$.

A subset $I$ of a $\Gamma$-ring $M$ is a right (left) $\Gamma$-ideal of $M$ if $I$ is an additive subgroup of $M$ and $I \Gamma M=$ $\{a \alpha c, a \in \mathrm{I}, \alpha \in \Gamma, c \in M\}(M \Gamma I)$ is contained in $I$. If $I$ is both a left and a right $\Gamma$-ideal, then we say that $I$ is an $\Gamma$-ideal or two sided $\Gamma$-ideal or simply ideal of $M$. Let $M$ be a $\Gamma$-ring and let $I$ be a subset of $M$. Define $l(I)=\{a \in M: a \Gamma I=0\}$ and $r(I)=\{a \in M: I \Gamma a=0\} . l(I)$ is called a left annihilator and $r(I)$ is called a right annihilator. The set $\{a \in M: a \Gamma I=I \Gamma a=0\}$ is called the annihilator of $I$ and is denoted by $A n n(I)$. If $I$ is an ideal of $M$, then $l(I), r(I)$ and $A n n(I)$ are left ideal, right and two sided ideal or simply ideal respectively.

An additive mapping $d: M \rightarrow M$ is called a derivation on $M$ if $d(x \alpha y)=d(x) \alpha y+x \alpha d(y)$ for all $x$, $y \in M, \alpha \in \Gamma$. An additive mapping $D$ of $M$ into itself is called a generalized derivation of $M$, with associated derivation $d$, if there is a derivation $d$ of $M$ such that $D(x \alpha y)=D(x) \alpha y+x \alpha d(y)$ for all $x$, $y \in M, \alpha \in \Gamma$. Obviously this notion covers the notion of a derivation (in case $D=d$ ) and a left centralizer (in case $d=0$ ). An additive mapping $D: M \rightarrow M$ is called a left centralizer if $D(x \alpha y)=$ $D(x) \alpha y$ for all $x, y \in M, \alpha \in \Gamma$.

Definition 2.1. Let $M$ be a $\Gamma$-ring. Derivations $d$ and $g$ on $M$ are said to be orthogonal if $d(x) \Gamma M \Gamma g(y)=0=g(y) \Gamma M \Gamma d(x)$ for all $x, y \in M$.

Let $M$ be a $\Gamma$-ring. An additive mapping $D: M \rightarrow M$ is said to be a generalized derivation if there exists a derivation $d: M \rightarrow M$ such that $D(x \alpha y)=D(x) \alpha y+x \alpha d(y)$ for all $x, y \in M, \alpha \in \Gamma$.

Two additive maps $d, g: M \rightarrow M$ are called orthogonal if $d(x) \alpha M \beta g(y)=0=g(y) \alpha M \beta d(x)$ for all $x, y \in M, \alpha, \beta \in \Gamma$.

Definition 2.2. Two generalized derivations $(D, d)$ and $(G, g)$ of $M$ are called orthogonal if $D(x) \alpha M \beta G(y)=0=G(y) \alpha M \beta D(x)$ for all $x, y \in M, \alpha, \beta \in \Gamma$.

The following two lemmas are due to [15] which are need to prove our main results.

Lemma 2.3. ([3], Lemma 2.1). Let $M$ be a 2-torsion free semiprime $\Gamma$-ring, $I$ be a nonzero ideal of $M$ and $a, b$ be the elements of $M$. Then the following conditions are equivalent.

(i) $a \alpha x \beta b=0$ for all $x \in I, \alpha, \beta \in \Gamma$.

(ii) $b \alpha x \beta a=0$ for all $x \in I, \alpha, \beta \in \Gamma$.

(iii) $a \alpha x \beta b+b \alpha x \beta a=0$ for all $x \in I, \alpha, \beta \in \Gamma$.

Moreover, if one of the three conditions is fulfilled and $l(I)=0$, then $a \alpha b=b \alpha a=0$. 
Lemma 2.4. ([3], Lemma 2.2). Let $M$ be a semiprime $\Gamma$-ring and $I$ be a nonzero ideal of $M$. Suppose that additive mappings $D$ and $G$ of $M$ into itself satisfy $D(x) \Gamma I \Gamma G(x)=0$ for all $x \in I$. Then $D(x) \Gamma I \Gamma G(y)=0$ for all $x, y \in I$.

\section{Orthogonality with Generalized Derivations on Semiprime $\Gamma$-rings}

We prove first some lemmas which will be frequently used to prove our results.

Lemma 3.1. Let $(D, d)$ and $(G, g)$ be two generalized derivations of $M$ and $l(I)=0$. If $D(I) \Gamma I \Gamma G(I)$ $=0$, then $D(M) \Gamma M \Gamma G(M)=0$.

Proof. Let $x, y, z \in M, r, s, t \in M$ and $\alpha, \beta \in \Gamma$. Then $0=D(x) \alpha z \beta G(y)=G(y) \alpha z \beta D(x)$ for all $x, y, z \in I$ , $\alpha, \beta \in \Gamma$ and Lemma 2.3, we have $0=D(x) \alpha g(r)=g(r) \alpha D(x)$ and by $g(r) \alpha D(x)=0$, we get $0=g(r) \alpha d(s)=d(s) \alpha g(r)$. Using these relations, we have $D(s) \alpha x \beta g(r)=0$ and so $0=$ $D(x \beta z) \alpha G(y)$, we obtain $d(z) \alpha G(y)=0$. Therefore $0=D(r \beta x) \alpha G(s \delta y)=D(r) \beta x \alpha G(s) \delta y$, which shows $D(r) \beta x \alpha G(s)=0$. Replace $x$ by $r_{0} \lambda G(s) \delta x \alpha D(r) \beta r_{0}$ for some $r_{0} \in M$, we have $D(r) \alpha r_{0} \delta G(s)=$ 0 , as desired.

Moreover, we have the following:

Lemma 3.2. Let $(D, d)$ and $(G, g)$ be two generalized derivations of $M$ and $I$ be an ideal of $M$ such that $l(I)=0$. Then the following conditions are equivalent.

(i) For any $x, y \in I, \alpha \in \Gamma$, the following relations hold:

(a) $D(x) \alpha G(y)+G(x) \alpha D(y)=0$.

(b) $\quad d(x) \alpha G(y)+g(x) \alpha D(y)=0$.

(ii) $D(x) \alpha G(y)=d(x) \alpha G(y)=0$ for all $x, y \in I, \alpha \in \Gamma$.

(iii) $D(x) \alpha G(y)=0$ for all $x, y \in I, \alpha \in \Gamma$ and $d G=d g=0$ for all $x, y \in \mathrm{I}, \alpha \in \Gamma$.

(iv) ( $D G, d g$ ) is a generalized derivation from $I$ to $M$ and $D(x) \alpha G(y)=0$ for all $x, y \in I, \alpha \in \Gamma$.

Proof. (i) $\Leftrightarrow$ (ii): By (a), (b) Lemmas 2.3 and 2.4, we have $0=D(x) \alpha z \beta G(y)=D(x) \alpha G(y)$ and using this $d(z) \alpha G(y)=0, x, y \in I, \alpha \in \Gamma$. This shows (ii). And the converse is easily obtained by the relations $D(x) \alpha G(y)=G(y) \alpha D(x)=0, x, y \in I, \alpha \in \Gamma$ and Lemma 2.3.

(ii) $\Rightarrow$ (iii) Let $x, y, z \in M, \alpha, \beta \in \Gamma$, then, by assumption, $D(x) \alpha z \beta G(y)=d(x) \alpha z \beta g(y)=0$. Then by Lemma 3.1, $d$ and $g$ are orthogonal, which shows $d g=0$. Moreover, by $0=d(x) \alpha G(y)$ and Lemma 2.3, we have, $0=d(d(r) \alpha s \beta G(y))=d(r) \alpha s \beta \mathrm{d} G(y)$ for $r, s \in M, \alpha, \beta \in \Gamma$. Taking $r=G(y)$, we have $d(G(y))=0$. Since $D(x) \alpha G(y)=G(y) \alpha d(x)=d(x) \alpha g(y)=0$, using Lemma 3.1, we obtain $d(G(r))=$ 0 , this gives (iii).

(iii) $\Rightarrow$ (iv) Let $x, y, z \in M, \alpha \in \Gamma$, then by $d G=d g=0$, we have $G(x) \alpha d(y)+d(x) \alpha g(y)=0=$ $g(x) \alpha d(y)+d(x) \alpha g(y)$. Then by the proof of (i) $\Rightarrow$ (ii), we see that $d(x) \alpha g(y)=0$ and so 
$G(x) \alpha d(y)=0$. Moreover, by $0=D(x) \alpha G(y)$, we have $D(x) \alpha g(z)=0$. Therefore $D G(x \alpha y)=$ $D G(x) \alpha y$ and thus $(D G, d g)=0$ is a generalized derivation from $I$ to $M$.

(iv) $\Rightarrow$ (ii) Let $x, y, z \in M, \alpha \in \Gamma,(D G, d g)$ is a generalized derivation if and only if

$G(x) \alpha d(y)+D(x) \alpha g(y)=0=d(x) \alpha g(y)+g(x) \alpha d(y)$,

So, we obtain $d g=0$. Furthermore by $0=D(x) \alpha G(y)$, we get $D(x) \alpha g(y)=0$ and by the above relation, we see $G(x) \alpha d(y)=0$. Therefore $G(x) \alpha z \beta d(y)=0$ and by Lemma 2.3 , we arrive at $d(y) \alpha G(x)=0$. This shows (ii).

Lemma 3.3 If $(D, d)$ and $(G, g)$ are two orthogonal generalized derivations of $M$, then the following relations hold.

(i) $D(x) \alpha G(y)=G(x) \alpha D(y)=0$, hence $D(x) \alpha G(y)+G(x) \alpha D(y)=0$ for all $x, y \in M, \alpha \in \Gamma$.

(ii) $d$ and $G$ are orthogonal, and $d(x) \alpha G(y)=G(y) \alpha d(x)=0$ for all $x, y \in M, \alpha \in \Gamma$.

(iii) $g$ and $D$ are orthogonal, and $g(x) \alpha D(y)=D(y) \alpha g(x)=0$ for all $x, y \in M, \alpha \in \Gamma$.

(iv) $d$ and $g$ are orthogonal derivations.

(v) $d G=G d=0$ and $g D=D g=0$.

(vi) $D G=G D=0$.

Proof. (i) By the hypothesis we have $D(x) \alpha z \beta G(y)=0$ for all $x, y \in M, \alpha \in \Gamma$. Hence we get

$D(x) \alpha G(y)=G(x) \alpha D(y)=0$ for all $x, y \in M, \alpha \in \Gamma$, by Lemma 2.3.

(ii), (iii) By $D(x) \alpha G(y)=0$ and $D(x) \alpha z \beta G(y)=0$ for all $x, y, z \in M, \alpha, \beta \in \Gamma$, we get

$0=D(r \delta x) \alpha G(y)=(D(r) \delta x+r \delta d(x)) \alpha G(y)=r \delta d(x) \alpha G(y)$ for all $r, x, y \in M, \alpha, \delta \in \Gamma$. Since $M$ is semiprime, $d(x) \alpha G(y)=0$ for all $x, y \in M, \alpha \in \Gamma$. Then we have

$d(x \delta r) \alpha G(y)=(d(x) \delta r+x \delta d(r)) \alpha G(y)=d(x) \delta r \alpha G(y)=0$ for all $r, x, y \in M, \alpha, \delta \in \Gamma$.

Therefore by Lemma 2.3, we obtain $G(y) \alpha d(x)=0$ for all $x, y \in M, \alpha \in \Gamma$, which shows (ii).

The proof of (iii) is similar.

(iv) We have

$0=D(x \delta z) \alpha G(y \beta w)=(D(x) \delta z+x \delta d(z)) \alpha(G(y) \beta w+y \beta g(w))$ for all $x, y, z, w \in M, \alpha, \beta, \delta \in \Gamma$.

by (i). Thus we get, $x \delta d(z) \alpha y \beta g(w)=0$ for all $x, y, z, w \in M, \alpha, \beta, \delta \in \Gamma$, by (ii) and (iii). Since $M$ is semiprime, we see that $d(z) \alpha y \beta g(w)=0$ for all $y, z, w \in M, \alpha, \beta \in \Gamma$, which shows that $d$ and $g$ are orthogonal.

(v), (vi) Using (ii) and (iv), we have

$0=G(d(x) \alpha z \beta G(y))=G d(x) \alpha z \beta G(y)+d(x) \alpha g(z \beta G(y))=G d(x) \alpha z \beta G(y)$ for all $x, y, z \in M, \alpha, \beta \in \Gamma$. Replacing $y$ by $d(x)$ in the above relation, we get $G d=0$ by the semiprimeness of $M$. Similarly, since each of $d(G(x) \alpha z \beta d(y))=0, D(g(x) \alpha z \beta D(y))=0, g(D(x) \alpha z \beta g(y))=0$ and $G(D(x) \alpha z \beta G(y))=$ 
0 holds for all $x, y, z \in M, \alpha, \beta \in \Gamma$, we have $d G=D g=g D=D G=G D=0$, respectively.

By Lemma 2.4, we get the following corollary:

Corollary 3.4 If $(D, d)$ and $(G, g)$ are two orthogonal generalized derivations of $M$, then $d g$ is a derivation and $(D G, d g)=(0,0)$ is a generalized derivation .

Lemma 3.5. Let $M$ be a semiprime $\Gamma$-ring. Let $I$ be an ideal of $\mathrm{M}$ and $V=A n n(I)$. If $(D, d)$ is a generalized derivation of $M$ such that $D(M), d(M) \subset I$, then $D(V)=d(V)=0$.

Proof. If $x \in V$, then $x \Gamma I=0$. By the hypothesis we have $d(I) \subset I$. Hence, $0=D(x) \alpha y+x \alpha d(y)=$ $D(x) \alpha y$ for all $y \in I, \alpha \in \Gamma$. Since $D(x) \in I \cap V$ and $M$ is semiprime, we get $D(x)=0$ for all $x \in V$. Similarly, we obtain $d(V)=0$.

Theorem 3.6 Let $(D, d)$ and $(G, g)$ be generalized derivations of $M$. Then the following conditions are equivalent.

(i) $(D, d)$ and $(G, g)$ are orthogonal.

(ii) For all $x, y \in M, \alpha \in \Gamma$, the following relations hold.

(a) $D(x) \alpha G(y)+G(x) \alpha D(y)=0$.

(b) $d(x) \alpha G(y)+g(x) \alpha D(y)=0$.

(iii) $D(x) \alpha G(y)=d(x) \alpha G(y)=0$ for all $x, y \in M, \alpha \in \Gamma$.

(iv) $D(x) \alpha G(y)=0$ for all $x, y \in M, \alpha \in \Gamma$ and $d G=d g=0$.

(v) $(D G, d g)$ is a generalized derivation and $\mathrm{D}(\mathrm{x}) \alpha \mathrm{G}(\mathrm{y})=0$ for all $\mathrm{x}, \mathrm{y} \in \mathrm{M}, \alpha \in \Gamma$.

(vi) There exist ideals $I$ and $V$ of $M$ such that:

(a) $I \cap V=0$ and $I \oplus V$ is an essential ideal of $M$.

(b) $D(M), d(M) \subset I$ and $G(M), g(M) \subseteq V$.

(c) $D(V)=d(V)=0$ and $G(I)=g(I)=0$.

Proof. (i) $\Rightarrow$ (ii), (iii), (iv) and (v) are proved by Lemma 2.4 and Corollary 3.4 .

(ii) $\Rightarrow$ (i) If we take $x \beta z$ instead of $x$ in (a), then by (b) we have

$$
0=D(x) \beta z \alpha G(y)+G(x) \beta z \alpha D(y) \text { for all } x, y, z \in M, \alpha, \beta \in \Gamma .
$$

Thus by Lemma 2.3 we arrive at $D(x) \Gamma M \Gamma G(y)=G(y) \Gamma M \Gamma D(x)=0$ for all $x, y \in M$.

(iii) $\Rightarrow$ (i) Since $0=(D(x) \beta z+x \beta d(z)) \alpha G(y)=D(x) \beta z \alpha G(y)$ for all $x, y, z \in M, \alpha, \beta \in \Gamma$, we get the result by Lemma 2.3 .

(iv) $\Rightarrow$ (i) Since $d g=0$, we have

$0=d G(x \alpha y)=d G(x) \alpha y+G(x) \alpha d(y)+d(x) \alpha g(y)+x \alpha d g(y)=G(x) \alpha d(y)$ for all $x, y \in M, \alpha \in \Gamma$. Thus $0=G(x) \alpha z \beta d(y)+x \alpha g(z) \beta d(y)=G(x) \alpha z \beta d(\mathrm{y})$ for all $x, y \in M, \alpha \in \Gamma$. Hence we get $d(y) \alpha G(x)=0$ for all $x, y \in M, \alpha \in \Gamma$, by Lemma 2.3. Then (i) follows from (iii).

(v) $\Rightarrow$ (i) Since ( $D G, d g$ ) is a generalized derivation, $d g$ is a derivation. Then we obtain 
$D G(x \alpha y)=D G(x) \alpha y+x \alpha d g(y)$ for all $x, y \in M, \alpha \in \Gamma$, and we have

$D G(x \alpha y)=D(G(x) \alpha y+x \alpha g(y))=D G(x) \alpha y+G(x) \alpha d(y)+D(x) \alpha g(y)+x \alpha d g(y)$ for all $x, y \in M, \alpha \in \Gamma$. Comparing the last two relations, we get $G(x) \alpha d(y)+D(x) \alpha g(y)=0$ for all $x, y \in M, \alpha \in \Gamma$. Since $D(x) \alpha G(y)=0$ for all $x, y \in M, \alpha \in \Gamma$, we get

$0=D(x) \alpha G(y \beta z)=D(x) \alpha G(y) \beta z+D(x) \alpha y \beta g(z)=D(x) \alpha y \beta g(z)$ for all $x, y, z \in M, \alpha, \beta \in \Gamma$.

Using (v), we have

$0=D(x) \alpha G(y \beta z)=D(x) \alpha G(y) \beta z+D(x) \alpha y \beta g(z)=D(x) \alpha y \beta g(z)$ for all $x, y, z \in M, \alpha, \beta \in \Gamma$. Hence we obtain $g(z) \alpha D(x)=0$ for all $x, z \in M, \alpha \in \Gamma$. Replacing $z$ by $y \beta z$ in the last relation we get $g(y) \beta z \alpha D(x)=0$ for all $x, y, z \in M, \alpha, \beta \in \Gamma$. Thus we have $D(x) \alpha g(y)=0$ for all $x, y \in M, \alpha \in \Gamma$. This implies that $G(x) \alpha d(y)=0$ for all $x, y \in M, \alpha \in \Gamma$, which shows that $d(y) \alpha G(x)=0$ for all $x, y \in M$, $\alpha \in \Gamma$. Therefore by (iii), we get the result.

(i) $\Rightarrow$ (vi) Let $I_{0}$ be the ideal of $M$ generated by $d(M) \cup D(M)$, let $A n n\left(I_{0}\right)=V$ and $A n n(V)=I$. By Lemma 2.4, we see that $D(x) \alpha G(y)=G(x) \alpha D(y)=0, d(x) \alpha G(y)=g(x) \alpha D(y)=0$ and $d(x) \alpha g(y)=$ $g(y) \alpha d(x)=0$ for all $x, y \in M, \alpha \in \Gamma$. Since $D(M), d(M) \subset I_{0}$ we obtain $G(M), g(M) \subset V$. Moreover by Lemma 3.5 and $I_{0} \subset I$ we have $D(V)=d(V)=0$ and $G(I)=g(I)=0$. Since $M$ is semiprime, $I \oplus$ $V$ is an essential ideal of $M$, which shows (vi).

Theorem 3.7. Let $(D, d)$ and $(G, g)$ be generalized derivations of $M$ and $I$ be a nonzero ideal of $M$ such that $l(I)=0$. Then the following conditions are equivalent.

(i) $(D, d)$ and $(G, g)$ are orthogonal.

(ii) For all $x, y \in I$, the following relations hold.

(a) $D(x) \alpha G(y)+G(x) \alpha D(y)=0$. (b) $d(x) \alpha G(y)+g(x) \alpha D(y)=0$.

(iii) $D(x) \alpha G(y)=d(x) \alpha G(y)=0$ for all $x, y \in I, \alpha \in \Gamma$.

(iv) $D(x) \alpha G(y)=0$ for all $x, y \in I, \alpha \in \Gamma$ and $d(G(x))=d(g(x))=0$ for all $x, y \in I$.

(v) $(D G, d g)$ is a generalized derivation on $I$ and $D(x) \alpha G(y)=0$ for all $x, y \in I, \alpha \in \Gamma$.

Proof. (i) $\Rightarrow$ (ii), (iii), (iv) and (v) are clear. Since (ii), (iii), (iv) and (v) are equivalent by Lemma 3.2, we assume (iii). This implies that $0=(D(x) \alpha z+x \alpha d(z)) \beta G(y)=D(x) \alpha z \beta G(y)$. Then we have $D(I) \Gamma I \Gamma G(I)=0$. Thus by Lemma 2.5 , we have by Theorem 3.6 , (iii) $\Rightarrow$ (i).

Theorem 3.8. If $(D G, d g)$ is a generalized derivations on $I$ and $l(I)=0$ then $(D G, d g)$ is a generalized derivations on $M$.

Proof. It is easily seen that $(D G, d g)$ is a generalized derivations on $I$ if and only if $G(x) \alpha d(y)+$ $D(x) \alpha g(y)=0, d(x) \alpha g(y)+g(x) \alpha d(y)=0$ for all $x, y \in I, \alpha \in \Gamma$. Then by the second relation, we have $d$ and $g$ are orthogonal. By the first relation $0=G(x) \alpha d(y)+D(x) \alpha g(y)$, we get $0=$ $G(x) \alpha z \beta d(y)+D(x) \alpha z \beta g(y)$, for all $x, y \in I, \alpha, \beta \in \Gamma$. Hence replacing $z$ by $g(y) \delta z$ in this relation and 
using the orthogonality of the derivations $d$ and $g$, we obtain $0=D(x) \alpha g(y) \delta z \beta g(y)$ which implies that $D(x) \alpha g(y)=G(x) \alpha d(y)=0$. Moreover by $0=D(x) \alpha g(y \delta r)$, we get $0=D(x) \alpha g(r)$ for all $r \in M$, $\alpha \in \Gamma$. Using this relation we have $D(s) \alpha x \delta g(r)=0$ and similarly we can see that $D(s) \alpha g(r)=$ $G(s) \alpha \mathrm{d}(r)=0$. Thus we obtain $(D G)(r \alpha s)=(D G)(r) \alpha s$ for all $r, s \in M, \alpha \in \Gamma$, which completes the proof.

Theorem 3.9 Let $(D, d)$ and $(G, g)$ be generalized derivations of $M$. Then the following conditions are equivalent.

(i) $(D G, d g)$ is a generalized derivation.

(ii) $(G D, g d)$ is a generalized derivation.

(iii) $D$ and $g$ are orthogonal, and $G$ and $d$ are orthogonal.

Proof. (i) $\Rightarrow$ (iii) Assume that ( $D G, d g$ ) is a generalized derivation. Thus, as in the proof of the Theorem 3.6, (v) $\Rightarrow$ (i) we obtain $G(x) \alpha d(y)+D(x) \alpha g(y)=0$ for all $x, y \in M, \alpha \in \Gamma$. Replacing $y$ by $y \beta z$ in the above relation, where $z \in M, \beta \in \Gamma$, we get $G(x) \alpha y \beta d(z)+D(x) \alpha y \beta g(z)=0$ for all $x$, $y \in M, \alpha, \beta \in \Gamma$. Since $(D G, d g$ ) is a generalized derivation, $d g$ is a derivation. Therefore $d$ and $g$ are orthogonal. Thus we have $0=G(x) \alpha g(z) \beta y \delta d(z)+D(x) \alpha g(z) \beta y \delta g(z)=D(x) \alpha g(z) \beta y \delta g(z)$ for all $x$, $y, z \in M, \alpha, \beta, \delta \in \Gamma$. Hence we get $D(x) \Gamma g(z) \Gamma M \Gamma D(x) \Gamma g(z)=0$ for all $x, z \in M$. By the semiprimeness of $M$, we obtain $D(x) \alpha g(z)=0$ for all $x, z \in M, \alpha \in \Gamma$. Thus $D(x) \alpha y \beta g(z)=0$ for all $x$, $y, z \in M, \alpha, \beta \in \Gamma$, and we have $G(x) \alpha y \beta d(z)=0$ for all $x, y, z \in M, \alpha, \beta \in \Gamma$.

(iii) $\Rightarrow$ (i) Since $D$ and $g$ are orthogonal, we get $D(x) \alpha y \beta g(z)=0$ for all $x, y, z \in M, \alpha, \beta \in \Gamma$.

Substituting $r \delta x$ for $x$ in the last relation, we arrive at $0=D(r \delta x) \alpha y \beta g(z)=D(r) \delta x \alpha y \beta g(z)+$ $r \delta d(x) \alpha y \beta g(z)=r \delta d(x) \alpha y \beta g(z)$ for all $r, x, y, z \in M, \alpha, \beta, \delta \in \Gamma$. Hence $d(x) \alpha y \beta g(z)=0$ for all $x, y$, $z \in M, \alpha, \beta \in \Gamma$, by the semiprimeness of $M$. Thus, we conclude that $d g$ is a derivation. Moreover since $D(x) \alpha y \beta g(z)=0$ for all $x, y, z \in M, \alpha, \beta \in \Gamma$, we also get $D(x) \Gamma(g(z) \Gamma M \Gamma D(x)) \Gamma g(z)=0$ and so $D(x) \Gamma g(z)=0$ for all $x, z \in M$, by the semiprimeness of $M$. Similarly, since $G$ and $d$ are orthogonal, we have $G(x) \alpha d(y)=0$ for all $x, y \in M, \alpha \in \Gamma$. Thus we obtain $D G(x \alpha y)=D G(x) \alpha y+x \alpha d g(y)$ for all $x, y \in M, \alpha \in \Gamma$, which means that $(D G, d g)$ is a generalized derivation.

(ii) $\Rightarrow$ (iii) is proved in a similar way.

Theorem 3.10. Let $(D, d)$ and $(G, g)$ be generalized derivations of $M$ and $l(I)=0$. Then the following conditions are equivalent.

(i) $(D G, d g)$ is a generalized derivation on $I$.

(ii) $(G D, g d)$ is a generalized derivation on $I$.

(iii) $D$ and $g$ are orthogonal, and $G$ and $d$ are orthogonal.

Proof. The proof is clear by Theorem 3.8 and 3.9.

Corollary 3.11. Let $(D, d)$ be a generalized derivations of $\mathrm{M}$ and $l(I)=0$. If $\left(D^{2}, d^{2}\right)$ is a 
generalized derivation on $I$, then $d=0$.

Proof. The fact that $\left(D^{2}, d^{2}\right)$ is a generalized derivation on $I$ is implies that $d$ and $d$ are orthogonal. Therefore we get $d=0$ by the semiprimeness of $M$.

Corollary 3.12. Let $(D, d)$ be a generalized derivations of $M$ and $l(I)=0$. If $D(x) \alpha D(y)=0$ for all $x, y \in I, \alpha \in \Gamma$, then $D=d=0$.

Proof. By the hypothesis we have $0=D(x) \alpha D(y \beta z)=D(x) \alpha D(y) \beta z+D(x) \alpha y \beta d(z)=D(x) \alpha y \beta d(z)$ for all $x, y, z \in I, \alpha, \beta \in \Gamma$. In particular, we see that $d(z) \alpha D(x)=0$ for all $x, z \in I, \alpha \in \Gamma$, by Lemma 2.3. Replacing $x$ by $x \delta y$ in the last relation we get $0=d(z) \alpha D(x) \delta y+d(z) \alpha x \delta d(y)=d(z) \alpha x \delta d(y)$ for all $x, y, z \in I, \alpha, \delta \in \Gamma$. Hence we obtain $d(s) \Gamma M \Gamma d(r)=0$ for all $s, r \in M$. In particular $d(s) \Gamma M \Gamma d(s)=$ 0 for all $s \in M$. Thus $d=0$ by the semiprimeness of $M$. Then we have $0=D(x \alpha z) \beta D(y)=$ $D(x) \alpha z \beta D(y)$ for all $x, y, z \in I, \alpha, \beta \in \Gamma$. By Lemma 3.1, we arrive at $D(r) \Gamma M \Gamma D(s)=0$ for all $r$, $s \in M$. In particular, $D(r) \Gamma M \Gamma D(r)=0$ for all $r \in M$, which implies $D=0$, as desired.

\section{REFERENCES}

[1] Albas, E., On ideals and orthogonal generalized derivations of semiprime rings, Math. J. Okayama Univ., 49(2007), 53-58.

[2] Argac, N., Nakajima, A. and Albas, E., On orthogonal generalized derivations of semiprime rings, Turk J. Math. 28(2004), 185-194.

[3] Ashraf, M. and Jamal M. R., Orthogonal Derivations in $\Gamma$-rings, Advances in Algebra, 3, 1, 2010,1-6.

[4] Atteya, M. J., On generalized derivations of Semiprime rings, International Mathematical Journal of Algebra 4(2010), 12, 591-598.

[5] Barnes W. E., On the Г-rings of Nabusawa, Pacific J. Math., 18, 1966, 411-422.

[6] Bresar, M. and Vukman J., Orthogonal derivation and extension of a theorem of Posner, Radovi Mathemateki 5(1989), 237-246.

[7] Dey, K. K., Paul A. Ch. and Davvaz B., Tri-additive mappings in a left s-unital rings, International J of Pure and App Math 23,4(2005), 465-474.

[8] Dey, K. K., Paul A. Ch. and Davvaz B., Derivations on Lie Ideals of Prime $\Gamma$-Rings, Communications in Computer and Information Science (CCIS) 655(2017), 380 - 390.

[9] Dey, K. K., Paul, A. Ch. and Rakhimov I. S., Semiprime Gamma Rings with Orthogonal Reverse Derivations, International Journal of Pure and Applied Math., 83(2) (2013), 233-245

[10] Dey, K. K., Paul, A. Ch. and Rakhimov I. S., Generalized derivations in semiprime gamma-rings, International Journal of Math. and Math. Sci., 2012, doi:10.1155/2012/270132

[11] Dey, K. K. and Paul, A. Ch., Generalized Derivations of Prime Gamma Rings, GANIT J. of Bangladesh Math. Soc. 33, 2013, 33-39

[12] Dey, K. K., Paul, A. Ch. and Rakhimov, I. S., Orthogonal Generalized Derivations in Semiprime Gamma Near-Rings, International Journal of Algebra, 6, 23(2012), 1127-1134.

[13] Hvala B., Generalized derivations in rings, Comm. Algebra, 26, 4(1998), 1147-1166.

[14] Nabusawa N, On a generalization of the Ring Theory, Osaka J. Math., 1(1964), 65-75.

[15] Suliman, N. N. and Majeed, A. R. H., Orthogonal derivations on an ideal of semiprime $\Gamma$-rings, Inter Math Forum 7, 28(2012), 1405-1412. 